\title{
NOUVELLE
}

\section{Inositol phosphatase et masse osseuse}

\section{Rôle d'INPP4b}

Jean Vacher
Institut de recherches cliniques de Montréal ; département de médecine, université de Montréal ; Department of Medicine, McGill University, 110, avenue des Pins Ouest, Montréal, Québec, Canada. vacherj@ircm.qc.ca
> La voie de signalisation de la phosphatidyl inositol 3 kinase (PI3K) participe à de nombreux mécanismes de régulation comme la croissance, la différenciation et la survie cellulaires [1]. Plus particulièrement, plusieurs études ont mis en évidence le rôle dans la physiologie osseuse de la PI3K et celui de trois inositol phosphatases qui agissent comme antagonistes de son mécanisme d'action [2-4] (Figure 1). Pten, un gène suppresseur de tumeur, code une inositol 5-phosphatase ayant une homologie avec la tensine, d'où son nom PTEN, phosphatase and tensin homologue, et qui est essentielle à la biologie de l'ostéoblaste. La perte de fonction de cette protéine dans les ostéoblastes entraîne une augmentation de leur survie et, par voie de conséquence, une masse osseuse accrue [5]. SHIP (Src homology 2 domain-containing inositol phosphatase) est une inositol 5-phosphatase à domaine $\mathrm{SH} 2$ et son ablation chez la souris induit un phénotype ostéoporotique par la stimulation de la fonction de résorption des ostéoclastes [6]. L'inositol polyphosphate 4-phosphatase, INPP4b, s'est révélé exercer une activité de suppresseur de tumeurs chez l'humain dans les cancers du sein, de la prostate et des ovaires [7], toutefois nous avons découvert très récemment un rôle majeur de cette phosphatase dans les cellules osseuses [8].

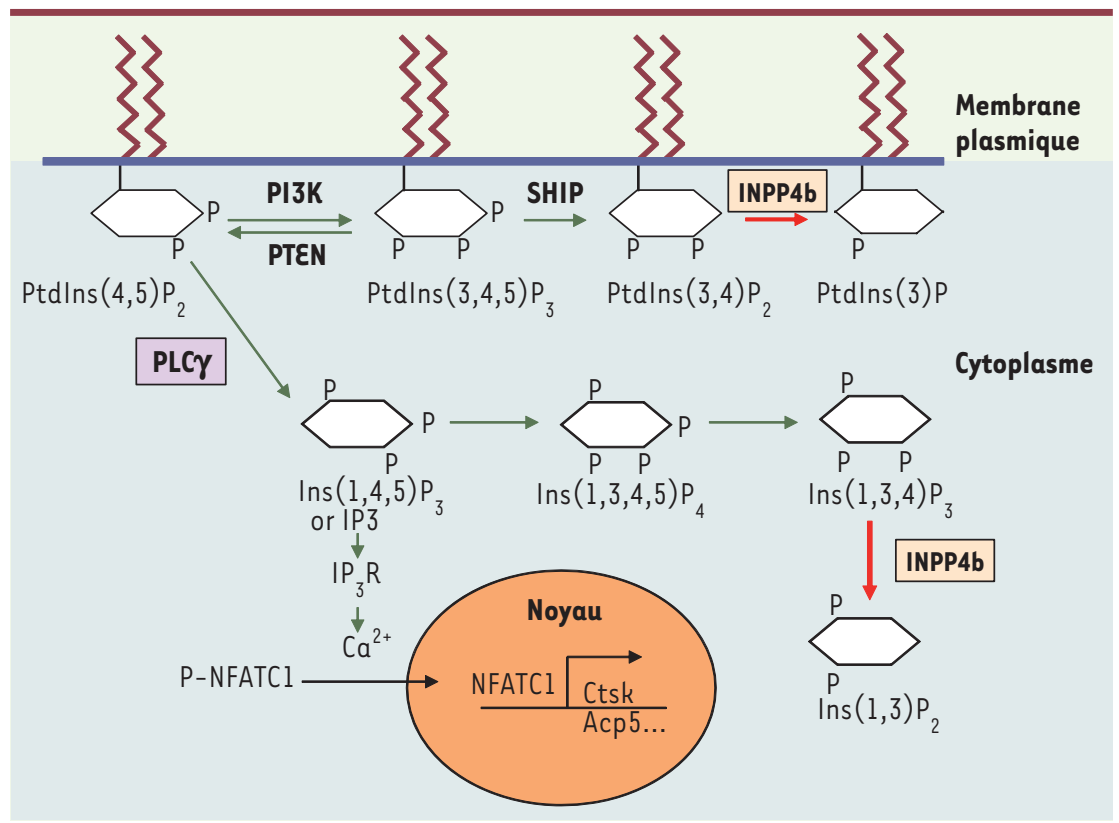

Figure 1. Voies de signalisation modulées par INPP4b. Représentation shématique des voies métaboliques des phosphatidylinositol et inositol phosphates indiquant le rôle potentiel d'INPP4b dans la signalisation de I'IP $P_{3}$, du calcium et du facteur transcriptionnel NFATCl essentiel pour la différenciation de l'ostéoclaste.

\section{INPP4b, un modulateur} de l'ostéoclastogenèse ex vivo et in vivo Nous avons isolé et caractérisé le gène codant pour la phosphatase INPP4b chez la souris [9]. Le rôle de cette phosphatase dans l'ostéoclastogenèse a été étudié tout d'abord dans la lignée monocytaire RAW qui peut se différencier en ostéoclastes matures. La surexpression d'INPP4b dans les cellules RAW entraîne une diminution du nombre d'ostéoclastes matures mettant en évidence l'influence négative de la phosphatase sur la différenciation ostéoclastique. À l'inverse, l'expression d'une protéine INPP4b inactive résulte en une augmentation significative de la différenciation et du nombre d'ostéoclastes (caractérisés en outre par une plus grande taille), ce qui démontre l'importance de l'activité catalytique dans la fonction de la protéine. Ainsi, ces résultats suggèrent une fonction additionnelle pour INPP4b, peut-être via les réarrangements du cytosquelette de l'ostéoclaste, qui est essentielle à son rôle dans la résorption de la matrice osseuse [11].

Nous avons ensuite analysé le rôle d'INPP4b in vivo dans un contexte physiologique, cellulaire et moléculaire en réalisant l'ablation génique d'Inpp $4 b$ chez la souris. Les souris homozygotes nulles pour le gène Inpp $4 b$ sont viables et leur développement semble normal. Cependant, une analyse phénotypique approfondie met en évidence une diminution marquée de la masse osseuse chez ces souris, corrélée à une augmentation de la population d'ostéoclastes. Ces souris développent un phénotype ostéoporotique. De plus, il existe une augmentation de la population d'ostéo- 
blastes, suggérant que l'absence d'INPP4b n'affecte pas le couplage ostéoclaste/ ostéoblaste. Ces résultats démontrent qu'INPP4b est un régulateur négatif de l'ostéoclastogenèse ex vivo et in vivo.

INPP4b module la voie de signalisation du calcium et de NFATCI dans l'ostéoclaste Afin de définir la voie de signalisation potentiellement modulée par INPP4b, nous avons analysé plusieurs voies intracellulaires: MAPKinases ( $p 38$, JNK, ERK1/2), AKT, NF-kB, NFATCl (nuclear factor of activated T-cells, cytoplasmic 1) ainsi que la phospholipase $C$ gamma $(P L C \gamma)$. Les effecteurs NF-KB et Akt sont faiblement stimulés, et il n'y a pas d'activation de la PLC $\gamma$ qui n'est donc pas directement régulée par INPP4b. En revanche, la voie de NFATCl est fortement stimulée en l'absence d'une protéine INPP4b active (surexpression de la protéine inactive dans les cellules RAW et utilisation d'ostéoclastes $\ln p p 4 b^{-/-}$ dépourvus de la protéine).

L'implication du facteur transcriptionnel NFATCl a été aussi corrélée à une augmentation de l'oscillation du calcium qui correspond à une libération dans le cytoplasme du calcium présent dans le réticulum endoplasmique [12]. En présence d'une concentration accrue de calcium cytosolique, la phosphatase calcineurine est activée et peut déphosphoryler NFATCl. Cette déphosphorylation permet la relocalisation de NFATCl dans le noyau où il agit comme facteur transcriptionnel. II en résulte une augmentation de la transcription de nombreux gènes cibles de NFATCl (cathepsine K, intégrines alphav-bêta3, TRAP/Acp 5 [tartrate resistant acid phosphatase ou acid phosphatase 5]) et NFATCl lui-même) [10] tant dans les cellules RAW exprimant la protéine inactive que dans les ostéoclastes $\ln p p 4 b^{-/-}$. Cette stimulation de la voie NFATCl se traduit in vivo par une population d'ostéoclastes plus importante et une augmentation significative de la résorption osseuse chez les souris Inpp $4 b^{-/-}$qui sont ostéoporotiques.

\section{INPP4B est associé à une variabilité} de la masse osseuse chez l'humain Notre analyse génomique du gène Inpp $4 b$ a localisé le locus dans une région de synténie avec le chromosome 4 humain [9]. Or plusieurs études ont défini un lien génétique entre cette région et la densité osseuse. Cette observation et les résultats cellulaires obtenus chez la souris nous ont incités à explorer le possible rôle d'INPP4b dans le métabolisme osseux chez l'humain. Nous avons donc entrepris une approche de gène candidat. De nombreux marqueurs génomiques polymorphiques associés au locus INPP4B ont été sélectionnés et utilisés pour le génotypage de deux cohortes de femmes préménopausées. Cette analyse a pu définir trois polymorphismes INPP4B associés à une variabilité significative de la densité osseuse mesurée au niveau du fémur et de la colonne vertébrale dans les deux groupes d'échantillons. Ces résultats suggèrent fortement qu'INPP4B peut être associé à une variabilité de la masse osseuse chez l'humain. Elle pourrait représenter un nouveau marqueur de sensibilité à l'ostéoporose permettant d'identifier les sujets sains ayant une prédisposition à développer la maladie.

En résumé, cette étude a permis de caractériser un nouveau modulateur de la masse osseuse chez la souris et l'humain. Ces résultats ont un impact majeur sur notre compréhension fondamentale de la physiologie de l'ostéoclaste. De plus, ils pourront aussi se traduire par l'établissement d'une détection des sujets à risque de développer une ostéoporose, et l'application de mesures préventives. $\diamond$

Inositol phosphatase and bone mass: role of INPP $4 b$

\section{CONFLIT D'INTERÊTS}

L'auteur déclare n'avoir aucun conflit d'intérêts concernant les données publiées dans cet article.

\section{REMERCIEMENTS}

Ce travail a été réalisé grâce au soutien des Instituts de recherche en santé du Canada (IRSC) et du Conseil de recherches en sciences naturelles et en génie du Canada (CRSNG).

\section{RÉFÉRENCES}

1. Cantley LC. The phosphoinositide 3-kinase pathway. Science 2002 ; 296 : 1555-57.

2. Golden LH, Insogna KL. The expanding role of PI3kinase in bone. Bone $2004 ; 34: 3-12$

3. Munugalavadla V, Vemula S, Sims $\varepsilon C$, et al. The $p 85 \alpha$ subunit of class IA phosphatidylinositol 3-kinase regulates the expression of multiple genes involved in osteoclast maturation and migration. Mol Cell Biol $2008 ; 28: 7182-98$.

4. Guntur AR, Rosen CJ. The skeleton: a multi-functional complex organ. New insights into osteoblasts and their role in bone formation: the central role of PI3Kinase. J Endocrinol 2011 ; 211 : 123-30.

5. Liu X, Bruxvoort KJ, Zylstra CR, et al. Lifelong accumulation of bone in mice lacking Pten in osteoblasts. Proc Natl Acad Sci USA 2007 ; 104 : 2259-64.

6. Takeshita S, Namba N, Zhao JJ, et al. SHIP-deficient mice are severely osteoporotic due to increased numbers of hyper-resorptive osteoclasts. Nat Med $2002 ; 8: 943-9$.

7. Agoulnik IU, Hodgson MC, Bowden WA, Ittman, MM. INPP4B: the new kid on the PI3K block. Oncotarget $2011 ; 2$ : 32l-8.

8. Ferron M, Boudiffa M, Arsenault M, et al. Inositol polyphosphate 4-phosphatase B as a regulator of bone mass in mice and humans. Cell Metab 2011 ; 14 : 466-77.

9. Ferron M, Vacher J. Characterization of the murine Inpp4b gene and identification of a novel isoform. Gene 2006 ; 376 : 152-61.

10. Takayanagi H, Kim S, Koga T, et al. Induction and activation of the transcription factor NFATcl (NFAT2) integrate RANKL signaling in terminal differentiation of osteoclasts. Dev Cell $2002 ; 3: 889-01$.

11. Baron R. L'ostéoclaste et les mécanismes moléculaires de la résorption osseuse. Med Sci (Paris) $2001 ; 17: 1260-9$.

12. Combettes L, Dupont G. Approches expérimentale et de modélisation de la signalisation calcique dans les cellules. Med Sci (Paris) $2011 ; 27$ : 170-6.

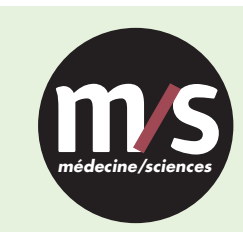

Tarifs d'abonnement $\mathrm{m} / \mathrm{s}-2012$

Abonnez-vous

à médecine/sciences
$>$ Grâce à $m / s$, vivez en direct les progrès des sciences biologiques et médicales

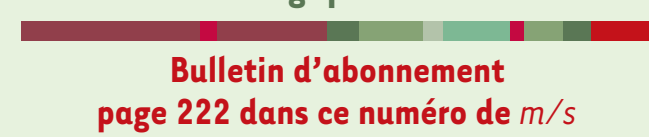

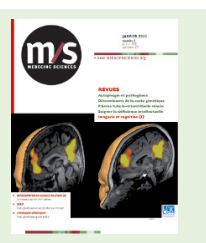

